\title{
Vaccine Evolution and Its Application to Fight Modern Threats
}

\author{
Emanuele Andreano 1,2,3, Ugo D'Oro ${ }^{2}$, Rino Rappuoli ${ }^{2,3,4}$ and Oretta Finco ${ }^{2 *}$ \\ ${ }^{1}$ Department of Life Sciences, University of Siena, Siena, Italy, ${ }^{2}$ GlaxoSmithKline, Siena, Italy, ${ }^{3}$ vAMRes Lab, Toscana Life \\ Sciences, Siena, Italy, ${ }^{4}$ Faculty of Medicine, Imperial College, London, United Kingdom
}

Before the development of the first vaccine, infectious diseases were a major cause of death around the globe with life expectancy estimated to be $<50$ years. Three measures have helped to drastically reduce the burden of infectious diseases but only vaccines have proven to be able to eradicate infectious agents. Herein, we describe new methodologies that have paved the way for what is currently known as modern vaccinology and the use of vaccines to tackle antimicrobial resistance, the biggest global threat of our time.

Keywords: vaccines, infectious diseases, antimicrobial resistance (AMR), vaccine development, vaccinology

OPEN ACCESS

Edited by:

Ennio Carbone,

Università degli Studi Magna Græcia

di Catanzaro, Italy

Reviewed by:

Sven Hammerschmidt,

University of Greifswald, Germany

Paola Italiani,

Italian National Research Council

(CNR), Italy

*Correspondence:

Oretta Finco

oretta.x.finco@gsk.com

Specialty section: This article was submitted to

Molecular Innate Immunity,

a section of the journal

Frontiers in Immunology

Received: 08 May 2019

Accepted: 09 July 2019

Published: 25 July 2019

Citation:

Andreano E, D'Oro U, Rappuoli R and Finco O (2019) Vaccine Evolution and

Its Application to Fight Modern

Threats. Front. Immunol. 10:1722.

doi: 10.3389/fimmu.2019.01722

\section{THE BURDEN OF INFECTIOUS DISEASES BEFORE ANTIBIOTICS AND VACCINE INTERVENTION}

Infectious diseases have always had a devastating impact on humankind. Some of the most catastrophic pandemics of our history include the Justinian plague (542-546 AD), which had a tragic toll of 100 million deaths, the bubonic plague (1347-50 AD), also known as the "Black Death," which erased one-third of the entire human population $(1,2)$, and more recently the "Spanish" influenza in 1918 which caused $\sim 50-100$ million deaths worldwide reducing the European population by half (3-5). Before the introduction of effective preventive and therapeutic strategies, life expectancy was estimated to be $<50$ years and bacterial infections were the imperative toll setting this limit (6). This scenario changed with the introduction of three measures that helped to dramatically reduce the death burden caused by infectious diseases. The measures include hygiene, antibiotics, and vaccination $(7,8)$. The introduction of penicillin in 1929 (9), and its first use in humans a decade later (10), led to a dramatic reduction of mortality caused by infectious diseases. Unfortunately, in 1940 the first case of a penicillin resistant E. coli strain was documented and by the late 1960 s over $80 \%$ of $S$. aureus strains acquired the same resistance (1012). Therefore, despite the use of antibiotics resulted to be an outstanding first line of defense to treat infections, pathogens have shown to quickly acquire resistance phenotypes after only few years from their introduction (13). Vaccines, on the other hand, have only rarely shown to induce resistant phenotypes as they usually aim to elicit a multi-targets immune response and their prophylactic use reduces the likelihood of spreading resistant-conferring mutations (14). Indeed the smallpox vaccine introduced in 1796, and subsequently manufactured from infected calf skin (15), has led to the eradication of this infectious agent in $1988(16,17)$. Therefore, despite the fact that antibiotics and vaccines are pivotal interventions against infectious diseases, vaccination has been the sole intervention capable of eradicating an infectious agent and, given its potential, it can also be considered as the most appropriate solution against future global threats represented by infectious diseases $(18-20)$. 


\section{REVERSE VACCINOLOGY AND THE DEVELOPMENT OF MODERN VACCINES}

Since Edward Jenner first vaccinated an 8 year old boy in 1796 by inoculating fresh cowpox lesion matter (21), enormous leaps forward have been made in the field of vaccine development. Empirical approaches like attenuation and inactivation of microorganisms were the first steps forward to modern vaccinology (22). Recently, new technologies such as glycoconjugates and the introduction of novel vaccine adjuvants changed the field of vaccines, however the biggest change came with the first sequencing of the Heamophilus influenzae whole genome in 1995, a discovery that allowed the birth of "Reverse Vaccinology," a genome-based approach to vaccine development $(23,24)$. This approach, following the sequencing and analysis of the Neisseria meningitidis serogroup B strain whole genome, allowed the identification of novel candidates and the development of a four-component meningococcus $B$ vaccine $(4 \mathrm{CMenB})(25,26)$. This recently licensed vaccine has already shown incredible effectiveness in the UK with $82.9 \%$ protection against all MenB strains in infants (27). The evolution of vaccine development further moved forward with the advancement of new methodologies and technological breakthroughs. Indeed, in 2016 the "reverse vaccinology 2.0" entered the stage. With this approach, the human immune system is analyzed at a single cell level allowing the characterization of the antibody response like never before (28). The gain of knowledge acquired by this approach allows to rapidly identify highly immunogenic antigens to develop novel and more efficacious vaccine candidates. The RSV fusion protein (F) case is a major example of the phenomenal power of the reverse vaccinology 2.0. Indeed, human B cells were directly isolated from RSV convalescent donors and cultured to naturally produce human monoclonal antibodies (humAbs). Among all the antibodies screened for RSV neutralization in vitro, the humAbs named D25 resulted in the most potent antibody with a median half-maximal inhibitory concentration (IC50) of $2.1 \mathrm{ng} / \mathrm{ml}$ (100-150 times more than palivizumab, the only monoclonal antibody approved by the FDA for RSV prevention in infants) (29). Interestingly, D25 was not capable of binding to the RSV F-protein in its post-fusion conformation, the only vaccine candidate available at the time against RSV (30). Then, McLellan and coworkers had the brilliant intuition to test D25 complex with the RSV F-protein to perform structural studies. This experiment was paramount in solving the crystal structure of RSV F-protein in its pre-fusion conformation (preF) which in turn led to the design of a stabilized RSV preF molecule $(30,31)$. Following the production of a soluble preF reagent, numerous human neutralizing antibodies have been identified allowing a deep characterization of the antigen surface and the identification of two preF-specific antigenic sites that have shown incredible high neutralization potency (32). The effectiveness of the preF antigen has already been proven in different animal models (mice, rhesus macaques, and calves) further supporting the potential of RSV preF as an ideal vaccine candidate against this pneumovirus $(10,13)$. The power of reverse vaccinology 2.0 has allowed, in $<5$ years since preF stabilization, to start clinical trials that are currently on-going to develop the first vaccine against RSV (7). This approach, which has found broad applicability to fight viral infections, could also be considered as a key stratagem to tackle bacterial infections.

\section{USE OF PEPTIDE-ANTIGEN DERIVED FOR GERMLINE TARGETING VACCINOLOGY}

The production of germline-targeting (GT) antigens for vaccine development is another pivotal example that underlies the outstanding potential of reverse vaccinology 2.0. Indeed, the combined knowledge acquired by the identification and characterization of novel antigens plus the functional/genetic analysis of human monoclonal antibodies naturally produced by infected or vaccinated human donors, can be used to design antigen-derived peptides, capable of tailoring the antibody immune response. In case of highly variable pathogens such as HIV, the use of the whole antigen can result in a strain specific response, while the development of GT-antigens can lead to the elicitation of broadly neutralizing antibodies (bnAbs) capable of clearing multiple infective strains. This is a two-step approach which, using different rationally designed immunogens, aims to: (1) prime the germline precursor B cell of antibodies previously shown to possess broadly neutralizing activity; (2) shepherding the bnAb population by driving their maturation affinity toward the highly immunogenic epitope of interest. GT-vaccinology has

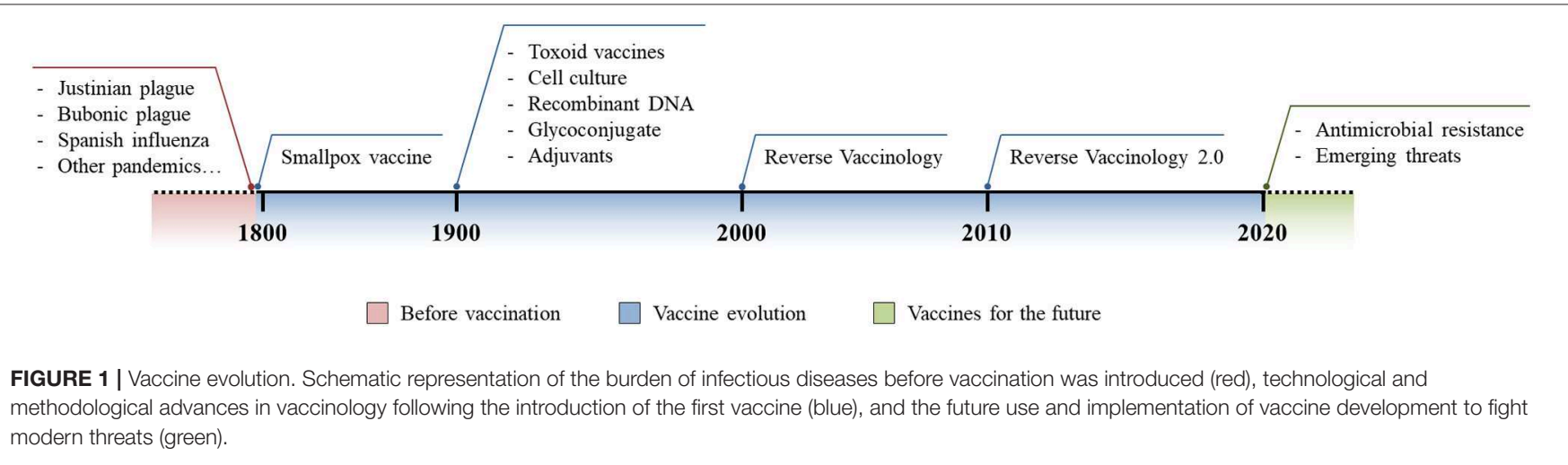


been used to elicit a specific class of HIV-1 gp120 CD4-binding site specific-bnAbs known as VRC01, through the use of engineered outer domain germline-targeting (eOD-GT) peptides (33). The interest to prime VRC01-bnAbs arises from their ability to mimic the CD4-binding to the gp120 receptor binding site and their capability to potently neutralize (median IC50 $40 \mathrm{ng} / \mathrm{mL}$ ) up to $98 \%$ of a large panel of global HIV-1 isolates $(34,35)$. An in-depth analysis of the VRC01 genetic features has shown peculiarities in this class of bnAbs. They classically derive from an extensively mutated (32-48\%) VH1-2*02 heavy chain germline which pairs with light chains, presenting a rare five amino acid long CDR3 motif (usually QQYEF) (36). These analyses were paramount for the development of novel and potentially therapeutic candidates to fight HIV infections. Examples of the use of VRC01-bnAbs as a therapeutic tool are the monoclonal antibodies named VRC-HIVMAB060-00-AB (VRC01) and a FCmodified version of this latter named VRC01LS. These two bnAbs are currently under clinical investigation (NCT02568215, NCT02716675, and NCT02599896) evaluating safety and efficacy in reducing acquisition of HIV-1 infection (37-40). In addition to monoclonal antibody development and application, the knowledge acquired from these studies and the ability to selectively expand this class of bnAbs upon immunization (41), have allowed the development of specific peptides as vaccine candidates capable of shepherding the immune system toward a VRC01-like antibody response. The most promising candidate is the tailored immunogen named eOD-GT8 60-subunit selfassembling nanoparticle (eOD-GT8 60mer) $(36,42)$ which has shown superior affinity and breadth of binding to germlinereverted VRC01-like bnAbs (41).

The HIV case described above further confirms the outstanding power of reverse vaccinology 2.0. Indeed, in only 3 years since its design and stabilization (43), the eOD-GT8 60mer antigen is under investigation in a phase I clinical trial in healthy adults aimed at assessing safety, tolerability and immunogenicity of this germline-targeting immunogen (NCT03547245).

\section{VACCINES FOR THE FUTURE: THE FIGHT AGAINST ANTIMICROBIAL RESISTANCE}

Despite antibiotics being the only lifesaving tool in fighting acute bacterial infection, as Stanley Falkow said (3), they are creating some problems of their own. In fact, the improper and excessive use of antibiotics has pressured bacteria to acquire antibiotic resistant phenotypes and this problem is currently growing out of control. Bacteria have shown several mechanisms to acquire antibiotic resistance and examples include the expression of $\beta$ lactamases, efflux pumps, modification of the cellular surface, and gene mutations to alter those molecules that are targeted by antibiotics (4). This phenomenon, known as antimicrobial resistance (AMR), is arguably one of the biggest threats that our world is facing today. Indeed, up to 700,000 deaths each year are AMR-related and these have been estimated to increase up to 10 million by 2050 , exceeding the 8.2 million deaths per year caused by cancer today $(8,44)$. A solution to this alarming threat would be the prevention of antibiotic resistant bacteria infections through vaccination, a strategy that has already proven its great value to humanity (6). Several reasons suggest that vaccines would be a promising solution against AMR. First, antibiotics have shown to rapidly become obsolete and resistance emerges soon after their introduction, while vaccines allow longlasting protection against infections and resistance has only rarely evolved after vaccination (13). Second, while antibiotics only hit a few metabolic target vaccines, based on the selected strategy, they can elicit a broad multi-target immune response reducing the probability of the evolution of resistant mutations. Furthermore, although major investments have been made to enrich antibiotic R\&D pipelines, the discovery of innovative antimicrobial targets are running dry since the 1970s. Therefore, given the incredibly high pace with which pathogens are capable of developing resistance to new classes of antibiotics, focusing our attention exclusively on antibiotic R\&D will not be sufficient $(13,45)$. In a marked contrast, thanks to incredible technological advancements of the last few decades, vaccine R\&D pipelines are promising for the development of innovative and highly effective vaccines which can have an important contribution in controlling $\operatorname{AMR}(13,18)$. Finally, antibiotics can only be used to treat individuals already infected, while successful vaccination campaigns can prevent the occurrence of infection, reducing the spread of the infectious agent and protecting the whole population through herd immunity $(8,20,46)$. Vaccine evolution has allowed us to address several unmet medical needs and, given all of the reasons stated above, it should be considered a key solution in fighting emerging threats such as AMR (Figure 1).

\section{CONCLUSIONS}

Since their introduction, vaccines have helped save billions of lives all over the world. Empirical approaches were not sufficient to support the development of vaccines against pathogens for which no preventive strategies or treatments were available. Methodological and technological advancements have introduced the world to modern vaccinology approaches which have unlocked the possibility to develop novel vaccines against virtually any pathogen. The RSV and HIV case studies reported herein, are clear examples of how innovative technologies and their corollary applications have paved the way for new experimental approaches capable of tackling and possibly addressing these unmet global medical needs. Vaccines have provided the basis for a global and sustainable public health in the past and they can potentially continue to do so by addressing major and upcoming global threats like AMR.

\section{AUTHOR CONTRIBUTIONS}

All authors listed have made a substantial, direct and intellectual contribution to the work, and approved it for publication.

\section{FUNDING}

This work has received funding under the European Research Council (ERC) advanced grant agreement number 787552 (vAMRes). 


\section{REFERENCES}

1. WHO. Chapter 3. WHO Report on Global Surveillance of Epidemic-Prone Infectious Diseases. (2000), 26p.

2. Schmid BV, Büntgen U, Easterday WR, Ginzler C, Walløe L, Bramanti B, et al. Climate-driven introduction of the Black Death and successive plague reintroductions into Europe. Proc Natl Acad Sci USA. (2015) 112:3020-5. doi: 10.1073/pnas.1412887112

3. Taubenberger JK, Morens DM. 1918 influenza: the mother of all pandemics. Emerg Infect Dis. (2006) 12:15-22. doi: 10.3201/eid1209.05-0979

4. Johnson NP, Mueller J. Updating the accounts: global mortality of the 1918-1920 "Spanish" influenza pandemic. Bull Hist Med. (2002) 76:105-15. doi: $10.1353 / \mathrm{bhm} .2002 .0022$

5. Morens DM, Taubenberger JK, Harvey HA, Memoli MJ. The 1918 influenza pandemic: lessons for 2009 and the future. Crit Care Med. (2010) 38(4 Suppl.):e10-e20. doi: 10.1097/CCM.0b013e3181ceb25b

6. Rappuoli R, Pizza M, Del Giudice G, De Gregorio E. Vaccines, new opportunities for a new society. Proc Natl Acad Sci USA. (2014) 111:12288-93. doi: 10.1073/pnas.1402981111

7. Rappuoli R. Twenty-first century vaccines. Philos Trans R Soc B Biol Sci. (2011) 366:2756-8. doi: 10.1098/rstb.2011.0075

8. Tagliabue A, Rappuoli R. Changing priorities in vaccinology: antibiotic resistance moving to the top. Front Immunol. (2018) 9:1068. doi: $10.3389 /$ fimmu.2018.01068

9. Fleming A. On the antibacterial action of cultures of a penicillium, with special reference to their use in the isolation of B. influenzae. Br J Exp Pathol. (1929) 10:226-36.

10. Abraham EP, Chain E. An enzyme from bacteria able to destroy penicillin. 1940. Rev Infect Dis. (1988) 10:677-8.

11. Lobanovska M, Pilla G. Penicillin's discovery and antibiotic resistance: lessons for the future? Yale J Biol Med. (2017) 90:135-45.

12. Lowy FD. Antimicrobial resistance: the example of Staphylococcus aureus. J Clin Investig. (2003) 111:1265-73. doi: 10.1172/JCI18535

13. Kennedy DA, Read AF. Why does drug resistance readily evolve but vaccine resistance does not? Proc Biol Sci. (2017) 284:20162562. doi: $10.1098 /$ rspb.2016.2562

14. Bloom DE, Black S, Salisbury D, Rappuoli R. Antimicrobial resistance and the role of vaccines. Proc Natl Acad Sci USA. (2018) 115:12868-71. doi: 10.1073/pnas.1717157115

15. Smith KA. Edward jenner and the small pox vaccine. Front Immunol. (2011) 2:21. doi: 10.3389/fimmu.2011.00021

16. Fenner F, Henderson DA, Arita I, Jezek Z, Ladnyi ID. Smallpox and Its Eradication. Geneva: WHO (1988).

17. Bhattacharya S. The World Health Organization and global smallpox eradication. J Epidemiol Commun Health. (2008) 62:909-12. doi: 10.1136/jech.2006.055590

18. Mishra RP, Oviedo-Orta E, Prachi P, Rappuoli R, Bagnoli F. Vaccines and antibiotic resistance. Curr Opin Microbiol. (2012) 15:596-602. doi: 10.1016/j.mib.2012.08.002

19. Jansen KU, Knirsch C, Anderson AS. The role of vaccines in preventing bacterial antimicrobial resistance. Nat Med. (2018) 24:10-9. doi: $10.1038 / \mathrm{nm} .4465$

20. Lipsitch M, Siber GR. How can vaccines contribute to solving the antimicrobial resistance problem? mBio. (2016) 7:e00428-16. doi: $10.1128 / \mathrm{mBio} .00428-16$

21. Riedel S. Edward Jenner and the history of smallpox and vaccination. Proc (Bayl Univ Med Cent). (2005) 18:21-5. doi: 10.1080/08998280.2005.11928028

22. Plotkin S. History of vaccination. Proc Natl Acad Sci USA. (2014) 111:12283-7. doi: 10.1073/pnas. 1400472111

23. Fleischmann RD, Adams MD, White O, Clayton RA, Kirkness EF, Kerlavage $\mathrm{AR}$, et al. Whole-genome random sequencing and assembly of Haemophilus influenzae Rd. Science. (1995) 269:496-512. doi: 10.1126/science.7542800

24. Rappuoli R. Reverse vaccinology. Curr Opin Microbiol. (2000) 3:445-50. doi: 10.1016/S1369-5274(00)00119-3

25. Vernikos G, Medini D. Bexsero $\AA$ chronicle. Pathog Global Health. (2014) 108:305-16. doi: 10.1179/2047773214Y.0000000162

26. Pizza M, Scarlato V, Masignani V, Giuliani MM, Arico B, Comanducci $\mathrm{M}$, et al. Identification of vaccine candidates against serogroup $B$ meningococcus by whole-genome sequencing. Science. (2000) 287:1816-20 doi: $10.1126 /$ science.287.5459.1816

27. Parikh SR, Andrews NJ, Beebeejaun K, Campbell H, Ribeiro S, Ward $\mathrm{C}$, et al. Effectiveness and impact of a reduced infant schedule of $4 \mathrm{CMenB}$ vaccine against group $\mathrm{B}$ meningococcal disease in England: a national observational cohort study. Lancet. (2016) 388:2775-82. doi: 10.1016/S0140-6736(16)31921-3

28. Rappuoli R, Bottomley MJ, D’Oro U, Finco O, De Gregorio E. Reverse vaccinology 2.0: human immunology instructs vaccine antigen design. J Exp Med. (2016) 213:469-81. doi: 10.1084/jem.201 51960

29. Kwakkenbos MJ, Diehl SA, Yasuda E, Bakker AQ, van Geelen CM, Lukens MV, et al. Generation of stable monoclonal antibody-producing B cell receptorpositive human memory B cells by genetic programming. Nat Med. (2010) 16:123-8. doi: $10.1038 / \mathrm{nm} .2071$

30. McLellan JS, Chen M, Leung S, Graepel KW, Du X, Yang Y, et al Structure of RSV fusion glycoprotein trimer bound to a prefusion-specific neutralizing antibody. Science. (2013) 340:1113-7. doi: 10.1126/science.12 34914

31. McLellan JS, Chen M, Joyce MG, Sastry M, Stewart-Jones GB, Yang $\mathrm{Y}$, et al. Structure-based design of a fusion glycoprotein vaccine for respiratory syncytial virus. Science. (2013) 342:592-8. doi: 10.1126/science.12 43283

32. Rossey I, McLellan JS, Saelens X, Schepens B. Clinical potential of prefusion RSV F-specific antibodies. Trends Microbiol. (2018) 26:209-19. doi: 10.1016/j.tim.2017.09.009

33. Jardine J, Julien JP, Menis S, Ota T, Kalyuzhniy O, McGuire A, et al. Rational HIV immunogen design to target specific germline B cell receptors. Science. (2013) 340:711-6. doi: 10.1126/science.12 34150

34. Huang J, Kang BH, Ishida E, Zhou T, Griesman T, Sheng Z, et al. Identification of a CD4-binding-site antibody to HIV that evolved near-pan neutralization breadth. Immunity. (2016) 45:1108-21. doi: 10.1016/j.immuni.2016. 10.027

35. Sok D, Burton DR. HIV Broadly neutralizing antibodies: taking good care of the 98. Immunity. (2016) 45:958-60. doi: 10.1016/j.immuni.2016.10.033

36. Jardine JG, Kulp DW, Havenar-Daughton C, Sarkar A, Briney B, Sok D, et al. HIV-1 broadly neutralizing antibody precursor B cells revealed by germline-targeting immunogen. Science. (2016) 351:1458-63. doi: 10.1126/science.aad9195

37. Cohen YZ, Caskey M. Broadly neutralizing antibodies for treatment and prevention of HIV-1 infection. Curr Opin HIV AIDS. (2018) 13:366-73. doi: 10.1097/COH.0000000000000475

38. Mayer KH, Seaton KE, Huang Y, Grunenberg N, Isaacs A, Allen $M$, et al. Safety, pharmacokinetics, and immunological activities of multiple intravenous or subcutaneous doses of an anti-HIV monoclonal antibody, VRC01, administered to HIV-uninfected adults: results of a phase 1 randomized trial. PLoS Med. (2017) 14:e1002435. doi: 10.1371/journal.pmed.1002435

39. Huang Y, Zhang L, Ledgerwood J, Grunenberg N, Bailer R, Isaacs A, et al. Population pharmacokinetics analysis of VRC01, an HIV-1 broadly neutralizing monoclonal antibody, in healthy adults. mAbs. (2017) 9:792-800. doi: 10.1080/19420862.2017.1311435

40. Gaudinski MR, Coates EE, Houser KV, Chen GL, Yamshchikov G, Saunders JG, et al. Safety and pharmacokinetics of the Fc-modified HIV-1 human monoclonal antibody VRC01LS: a phase 1 open-label clinical trial in healthy adults. PLoS Med. (2018) 15:e1002493. doi: 10.1371/journal.pmed.1002493

41. Abbott RK, Lee JH, Menis S, Skog P, Rossi M, Ota T, et al. Precursor frequency and affinity determine B cell competitive fitness in germinal centers, tested with germline-targeting HIV vaccine immunogens. Immunity. (2018) 48:133-46.e6. doi: 10.1016/j.immuni.2017.11.023

42. Briney B, Sok D, Jardine JG, Kulp DW, Skog P, Menis S, et al. Tailored immunogens direct affinity maturation toward HIV neutralizing antibodies. Cell. (2016) 166:1459-70.e11. doi: 10.1016/j.cell.2016.08.005

43. Jardine JG, Ota T, Sok D, Pauthner M, Kulp DW, Kalyuzhniy O, et al. HIV-1 VACCINES. Priming a broadly neutralizing antibody response to HIV-1 using a germline-targeting immunogen. Science. (2015) 349:156-61. doi: $10.1126 /$ science.aac5894 
44. The Review on Antimicrobial Resistance. Tackling Drug-Resistant Infections Globally: Final Report and Recommendations. (2016). Available online at https://amr-review.org/sites/default/files/160518_Final\%20paper_with \%20cover.pdf (accessed May 7, 2018).

45. O'Neil J. Tackling Drug-Resistant Infections Globally: Final Report and Recommendations. (2016). Wellcome Trust and UK Government.

46. Mallory ML, Lindesmith LC, Baric RS. Vaccination-induced herd immunity: successes and challenges. J Allergy Clin Immunol. (2018) 142:64-6. doi: 10.1016/j.jaci.2018.05.007
Conflict of Interest Statement: RR, OF, and UD'O are full-time employees of GSK group of companies. EA participated in a postgraduate program at GSK.

Copyright ( 2019 Andreano, D’Oro, Rappuoli and Finco. This is an open-access article distributed under the terms of the Creative Commons Attribution License (CC $B Y)$. The use, distribution or reproduction in other forums is permitted, provided the original author(s) and the copyright owner(s) are credited and that the original publication in this journal is cited, in accordance with accepted academic practice. No use, distribution or reproduction is permitted which does not comply with these terms. 